so ausgezeichneter Weise geschrieben, daß es unter den klassischen naturwissenschaftlichen Arbeiten nicht fehlen darf.

G. J.

Das Zeisswerk und die Karl Zeiss-Stiftung in Jena. Ihre wissenschaftliche, technische und soziale Entwicklung und Bedeutung für weitere Kreise dargestellt von Felix Auerbach. Mit 78 Abbildungen im Text. $8^{\circ}$. VI and 124 S.1903. Verlag von G. Fischer, Jena. M 2.-.

Professor Auerbach, ein Mann, welcher durch Freundschaft mit den Leitern des Zeisswerles verbunden, Gelegenheit hatte, das Emporblühen des Instituts viele Jahre hindurch aus nächster Nähe zu beobachten, hat uns dies Büchlein geschenkt. Er stellt in seiner Einleitung einen Vergleich voran, den zweier Unternehmungen, deren jede auf ihrem Gebiete eine fährende Weltstellung einnimmt: Siemens und Halske in Berlin und Kärl Zeiss in Jena. "Hervorgegangen aus bescheidenen, mechanischen Werkstätten - Halske in Berlin, Zeiss in Jena - deren Tätigkeit sich sozusàgen über ihr StraRenviertel nicht hinausstreckte, sind sie beide durch den Eintritt eines der Znnft fremden Mannes - dort des Artillerieoffiziers Werner Siemens, bier des Universitätsdozenten Ernst Abbe, befruchtet worden und aus dem Straßenviertel ist dor Erdball geworden“. Wäbrend aber Werner Siemens in seinen „Lebenserinnerungen" nicht nur eine Selbstbiographie snndern anch eine Entwicklungsgeschichte seines Unternehmens hinterlassen hat, blieb es hier einem Aufenstehenden vorbehalten den Werdegang des führenden optischen Instituts zu beschreiben. Was dadurch vielleicht an Unmittelbarkeit, an der Schilderung der Verknüpfung persönlicher Erlebnisse mit dem Entstehen der Werke verloren gegangen ist, das gewinnt anderseits diese Darstellung an Sachlichkeit und Objektivität. Und wer immer Interesse hat an der außerordentlichen culturellen Entwicklung Deutschlands der letzten Jahrzehnte, der wird das Büchlein. mit seinen Schilderungen, nicht nur der größten feinmechanischoptischen Betriebe, sondern auch seiner wunderbaren, von idealem Geiste geschaffenen und getragenen sozialen Verhältnisse aller werktätig Beteiligten mit ungeteiltem großen Vergnügen lesen.

St. $M$.

\title{
Vierstellige Tafeln und Gegentafeln für logarithmisches
} und trigonometrisches Rechnen in zwei Farben zusammengestellt von Dr. Hermann Schubert, Professor an der Gelehrtenschule des Johanneums. Zweite Auflage (Sammlung Göschen). $128 \mathrm{~S} .12^{0}$. G. J. Göschen, Leipzig 1903. Ladenpreis geb. M -.80.

Die vorliegende zweite Auflage dieses praktischen Büchleins unterscheidet sich nur in der äußeren Ausführung von der ersten Auflage, die im X. Jahrgang der "Monatshefte" (Literaturberichte S. 5) mit ausführlicher Inhaltsangabe besprochen wurde. Während in der ersten Auflage die Logarithmen rot, alle übrigen Zahlen schwarz gedruckt waren, so sind in der neuen Auflage an Stelle dieser Farben, die weniger grell sich unterscheidenden Farben braun nnd blau getreten. Wer rasch und sicher logarithmische Rechnungen ausführen will, wird sich mit Vorteil dieser Tafeln bedienen.

Tietze.

Wathematical papers of the late George Green edited by N. M. Ferrers, M. A. Facisimilé reprint. Paris. Librairie scientifique A. Hermann 1903. $8^{\circ}$. XII und $336 \mathrm{~S}$. Preis M 16.- 